\title{
The alteration of circulating exosomal mRNAs in acute myocardial infarction is associated with inflammation response mediated by neutrophils
}

\section{Guo-dong HE}

Research Department of Medical Sciences, Guangdong Provincial People's Hospital, Guangdong Academy of Medical Sciences, School of Medicine, South China University of Technology, Guangzhou, Guangdong, 510080, China

\section{Yu-qing HUANG}

Department of Cardiology, Guangdong Provincial Key Laboratory of Coronary Heart Disease Prevention, Guangdong Cardiovascular Institute, Guangdong Provincial People's Hospital, Guangdong Academy of Medical Sciences, School of Medicine, South China Universit

\section{Lin LIU}

Department of Cardiology, Guangdong Provincial Key Laboratory of Coronary Heart Disease Prevention, Guangdong Cardiovascular Institute, Guangdong Provincial People's Hospital, Guangdong Academy of Medical Sciences, School of Medicine, South China Universit

Jia-yi HUANG

Department of Cardiology, Guangdong Provincial Key Laboratory of Coronary Heart Disease Prevention, Guangdong Cardiovascular Institute, Guangdong Provincial People's Hospital, Guangdong Academy of Medical Sciences, School of Medicine, South China Universit

\section{Kenneth Lo}

Department of Epidemiology, Centre for Global Cardiometabolic Health, Brown University, Providence, USA

\section{Yu-ling YU}

Department of Cardiology, Guangdong Provincial Key Laboratory of Coronary Heart Disease Prevention, Guangdong Cardiovascular Institute, Guangdong Provincial People's Hospital, Guangdong Academy of Medical Sciences, School of Medicine, South China Universit

\section{Chao-lei CHEN}

Department of Cardiology, Guangdong Provincial Key Laboratory of Coronary Heart Disease Prevention, Guangdong Cardiovascular Institute, Guangdong Provincial People's Hospital, Guangdong Academy of Medical Sciences, School of Medicine, South China Universit

\section{Bin ZHANG}

Department of Cardiology, Guangdong Provincial Key Laboratory of Coronary Heart Disease Prevention, Guangdong Cardiovascular Institute, Guangdong Provincial People's Hospital, Guangdong Academy of Medical Sciences, School of Medicine, South China Universit 


\section{Research}

Keywords: acute myocardial infarction, exosomes, mRNAs, acute inflammatory response, neutrophil

Posted Date: May 3rd, 2020

DOl: https://doi.org/10.21203/rs.3.rs-25385/v1

License: (9) This work is licensed under a Creative Commons Attribution 4.0 International License. Read Full License 


\section{Abstract}

Background Although many cardiovascular disease studies have focused on the characteristics of microRNAs in circulating exosomes, the profile and the potential clinical diagnostic value of plasma exosomal long RNAs (exoLRs) in acute myocardial infarction (AMI) is still unknown.

Methods In this study, exosomes isolation and RNA sequencing were applied to achieve the circulating exoLRs profile of $10 \mathrm{AMI}$ patients, 8 stable coronary artery disease (CAD) patients, and 10 healthy individuals. Bioinformatics approaches were used to investigate the features and potential clinical value of exoLRs.

Results Each sample from $2 \mathrm{~mL}$ of plasma could reliably achieve more than 8000 exosomal messenger RNAs (mRNAs) making up a majority of the total exoLRs. Immune cell types analyzed by CIBERSORT showed that neutrophils and monocytes were significantly enriched in the AMI group compared to healthy individuals and the CAD group which were consistent with the clinical baseline characteristics. Similarly, the biological processes enrichment analyses of different exosomal mRNAs and co-expression network analysis both indicated that neutrophils activation associated processes were also significantly enriched in the AMI group. We further identified two exosomal mRNA ALPL and CXCR2 which might be served as potential biomarkers with high diagnostic efficiency through co-expression network analysis and receiver operating characteristic curve (ROC).

Conclusions In summary, our study explored the alteration of exoLRs in the AMI patients which might associate with the acute inflammatory response mediated by neutrophils. We found that exosomal mRNAs ALPL and CXCR2 might be potentially useful for AMI diagnosis.

\section{Background}

Exosomes secreted by most cell types are a class of lipid membrane-enclosed extracellular vesicles ranging in size from 40 to $100 \mathrm{~nm}[1,2]$. These small vesicles not only contain proteins, lipids, RNAs, and metabolites of the source cell but also maintain the compositions stabilized[3]. Therefore, exosomes are now progressively considered as crucial mediators of cell-cell communication and promising potential biomarkers for the disease[4, 5].

So far, most studies have focused on examining changes of microRNAs in circulating exosomes and exosomal microRNAs in cardiovascular disease have been well characterized and studied[6]. However, the exosomal mRNAs may not be extensively applied because the production of microRNAs in exosomes is lack of specific small quantity and lack of specificity [7].

Circular RNA (circRNA), long noncoding RNAs (IncRNA), and messenger RNA (mRNA) known as long RNAs are present and stabilized in exosomes[8]. More and more evidence indicates that the exosomal mRNA may have functional and potential clinical implications[9]. Among various exosomal mRNAs from tubular epithelial cells to macrophages, CCL2 is crucial for albumin-Induced tubulointerstitial 
inflammation[10]. What's more, two serum exosomal mRNAs, KRTAP5-4 and MAGEA3 could be potential biomarkers to detect colorectal cancer[11]. However, very few studies have concentrated on the characteristics of exosomal long RNAs (exoLRs) in cardiovascular disease, especially for acute myocardial infarction (AMI) known as a common disease with serious consequences in mortality, morbidity, and cost to the society[12]. And the profile of circulating exoLRs in AMI patients remains unknown.

In this study, we explored the plasma exoLRs profile of AMI, stable coronary artery disease (CAD), and healthy individuals by RNA sequencing analyses to investigate its features and potential diagnostic value in clinical. The plasma exoLRs profile could not only reflect the relative fractions of circulating immune cell types but also distinguish patients with AMI from CAD and healthy individuals. Intriguing exosomal mRNAs were identified to serve as potential biomarkers for AMI diagnosis. It might contribute to promoting the investigation of the intrinsic mechanisms of AMI.

\section{Methods}

\section{Patients}

This is a case-control study. There were 10 AMI, 8 CAD patients and 10 healthy individuals were recruited at Guangdong Provincial People's Hospital from December 2018 to January 2019. The AMI and CAD patients were diagnosed by laboratory tests and coronary angiography according to the European Society of Cardiology (ESC) guidelines $[13,14]$. Participants aged between 18-75 years are included, regardless of gender.

The healthy individual's recruitment criteria of included: normal renal and liver function, no history of smoking, malignancy, recent cardiovascular or cerebrovascular event, rheumatological disorders, no diagnosis of chronic heart failure, diabetes, acute or chronic infectious disease, aortic dissection, pulmonary embolism, myocarditis or pericarditis, and congenital heart disease.

Ethical approval of human sample collection was achieved from the Ethics Committee of Guangdong Provincial People's Hospital (No.2018160A), and all patients provided informed consent.

\section{Extraction exosomal RNAs from serum and library construction, sequencing and data analysis}

$2 \mathrm{~mL}$ of venous blood from patients were collected into Ethylene Diamine Tetraacetic Acid (EDTA) routine blood tubes. Plasma was separated by centrifugation at $3000 \mathrm{rpm}$ for $10 \mathrm{~min}$ at $4^{\circ} \mathrm{C}$ and collected into cryogenic vials and stored at $-80^{\circ} \mathrm{C}$. The exoRNeasy Serum/Plasma kit (Qiagen) was applied to extract the exosomal total RNAs. According to the handbook of the kit, $1 \mathrm{~mL}$ plasma was used to isolate exosomal RNAs. SMART technology (Clontech) was applied to construct the RNA-seq libraries. Each RNA sample used for sequencing was performed on the illumine Nova-Seq 6000 System with technical support of Guangzhou Epibiotek Co., Ltd. HISAT2 was applied to align with the sequencing reads data 
[15]. GENCODE database was used to annotate mRNA and IncRNA. CircRNAs from unmapped reads were identified through Acfs[16].

\section{Measurement of exosome characterization}

The morphological characteristics of the exosomes were observed by transmission electron microscopy (TEM) and their size and distribution were measured by NanoSight NS500 (NanoSight Ltd, Amesbury, United Kingdom). Exosomal protein markers CD9 and CD63 were detected by western blotting.

\section{Different exosomal long RNA analysis and functional enrichment analysis}

NetworkAnalyst (http://www.networkanalyst.ca), a visual analytics platform for comprehensive gene expression profiling[17], was applied to identify different exoLRs between AMI and CAD/normal samples. RNAs with P-value $<0.01$ and $\log _{2}$ fold change $\geq|1|$ were considered as significantly different exoLRs. The "clusterProfiler" package was used to investigate Gene Ontology (GO) enrichment analysis including biological processes (BPs), cellular components (CCs), and molecular functions (MFs) with P-value $<0.05$ considered statistically significant[18].

\section{Co-expression network of exosomal mRNA}

Exosomal mRNAs would be firstly filtered if the expression values were less than $1 \mathrm{FPKM}$ in at least $90 \%$ samples. The remaining mRNAs with standard deviation greater than 0.2 were fed into an R package for weighted correlation network analysis[19].

Appropriate soft-threshold power was selected to ensure the co-expression network according to the scale-free topology. The weighted adjacencies and correlations were transformed into a topological overlap matrix (TOM), followed by calculating the corresponding dissimilarity (1-TOM). Next, 1-TOM as the distance measure was applied to a hierarchical clustering analysis of genes. A dynamic tree cut algorithm was used to identify modules. According to the correlations of module eigengene and clinical traits, significant modules were identified with P-value $<0.05$. Top 50 exosomal mRNAs connections based on topological overlap in significant module were used to construct a network diagram using Cytoscape[20] and the exosomal mRNA with eigengene connectivity $>0.8$ in the module related to clinical traits was considered as candidate hub genes[21].

\section{Statistical analysis}


Principal component analysis (PCA) was applied to evaluate the variables in the three groups with the $\mathrm{R}$ package. Continuous variables with normally distributed were displayed as mean with standard deviation (SD) and analyzed by the t-test. Otherwise, they would be displayed as medians and analyzed by the nonparametric test. Pearson's correlation coefficient was applied to correlations analysis between two variables. RNA expression levels were shown as mean with fragments per kilobase per million (FPKM). The area under the curves (AUC) of receiver operating characteristic (ROC) was applied to evaluate the specificity and sensitivity of the exosomal mRNA for AMI diagnosis. The PASW Statistics 18.0 software was applied to all statistical analyses. P-value $<0.05$ was considered statistically significant.

\section{Results}

\section{Clinical baseline characteristics of patients}

The clinical characteristics of the three groups (10 AMI, 8 CAD, and 10 controls) are presented in Table 1. Acute inflammation levels between CAD group and control group were similar, whereas the AMI group had significantly higher acute inflammation levels at study entry. CKMB and HBDH were also higher in AMI patients than the rest subjects.

\section{A brief view of the workflow of human plasma exosomal long RNA-seq and its characteristics in each group}

Reliable exosomal long RNA-seq data were obtained according to the process of plasma isolation, purification of exosomes, exosomes RNA extraction, and RNA-seq library construction (Figure 1A). Transmission electron microscopy results showed membrane-enclosed structures of exosomes without similar sizes and uniform distribution in a dark background (Figure 1B). The average diameter of the isolated exosomes was $75.83 \mathrm{~nm}$ measured by the NanoSight instrument (Figure 1C). Membrane markers of exosomes CD9 and CD63 were confirmed by western blots (Figure 1D). The mRNA constituted $58.46 \%$ of total mapped reads. Pseudogenes and circRNA accounted for $12.80 \%$ and $11.73 \%$, respectively, whereas IncRNA and antisense RNA were only $7.94 \%$ and $7.55 \%$, respectively (Figure 1E). What's more, we found the numbers of mRNA, circRNA, IncRNA, and pseudogenes in the AMI group were all significantly higher than the control group (Figure 1F).

\section{Exosomal long RNA may reflect relative fractions of immune cell types}

Twenty-two types of immunocyte from exosomal sequencing data of each sample were investigated through an established computational resource (CIBERSORT) (Figure 2A). The PCA of the immunological profile showed an un-uniform distribution (Figure $4 C$ ). Memory B cells were the lowest in the CAD group, whereas naïve $B$ cells of the CAD group were the highest. Neutrophils and monocytes were significantly 
enriched in the AMI group which were consistent with the clinical baseline characteristics. The results indicated that circulating exosomal long RNA may reflect the circulating immunological profile.

\section{Different exosomal long RNA analysis between the AMI and the control}

Compared to the control group, 296 different exosomal mRNAs consisting of 254 up- and 42 downregulated in the AMI group (Figure 3A). These 296 mRNAs with significant differences were visualized in Figure 3B. However, only a few different IncRNAs or circRNAs were identified (Figure 3C and 3D). To investigate the biological processes enrichment of different exosomal mRNAs, ClusterProfiler was used to analyze and visualize functional profiles (Gene Ontology) of 296 different exosomal mRNAs. Meanwhile, these different exosomal mRNAs were subjected to https://www.networkanalyst.ca for enrichment maps of biological processes. The results of functional profiles analysis were shown as a bar plot (Figure 3E) and the enrichment map of biological processes was shown as a network (Figure 3F). Among the top 10 biological processes in Figure 3E and Figure 3F, neutrophil degranulation, neutrophil activation, and neutrophil activation involved in immune response were significantly enriched. The enrichment map (Figure 3F) showed that the inflammatory response might be the core biological processes.

Above all, through the analysis of different exosomal long RNA between the AMI and the control, we found that circulating exosomal mRNAs in the AMI group might play a crucial role in acute inflammation response mediated by neutrophils.

\section{Different exosomal mRNA analysis between AMI and CAD}

When compared to the CAD group, 230 different exosomal mRNAs consisting of 120 up- and 110 downregulated in the AMI group (Figure $3 \mathrm{~A}$ ). The results of functional profiles analysis (Figure 4D) were shown that neutrophil activation played a leading role in biological processes. To further investigate the relationship between $\mathrm{AMI}$ and $\mathrm{CAD}$, two intersections of differently up- or down-regulated exosomal mRNAs among AMI, CAD, and control groups were shown in Figure $4 B$ and $4 C$. There were 35 different exosomal mRNAs (31 up- and 4 down-regulated) overlapped between the comparison of AMI and control and comparison of AMI and CAD. The results of gene ontology analysis of these 35 different exosomal mRNAs showed that myeloid leukocyte activation was mainly enriched (Figure 4E). Besides, we found that ALPL was special since it was the only one up-regulated exosomal mRNA among the three comparison sets (Figure 4B), which might serve as a potential biomarker for AMI diagnosis.

\section{Co-expression network analysis of exosomal mRNAs in AMI}


To further investigate the union of genes related to AMI, we applied WGCNA(weighted gene co-expression network analysis) to clarify the key modules and the highly correlated exosomal mRNAs in AMI. 43 exosomal mRNAs modules were identified by the hierarchical clustering dendrogram (Figure 5A). The association of the 43 co-expression modules was analyzed by the topological overlap matrix plot which consisted of the modules and the corresponding hierarchical clustering dendrogram (Figure 5B). For module-trait analyses, only the light-yellow module containing 175 exosomal mRNAs was related to AMI (Figure 5C). Although there was no significant enriched KEGG biological pathway in the light-yellow module, the results of $\mathrm{GO}$ enrichment analysis indicated that the light-yellow module was involved in inflammation respond mediated by neutrophils such as neutrophil aggregation and chemokine production (Figure 5D). The association between the light-yellow module and AMI could be also supported by enrichment analyses of different exosomal mRNAs between AMI (Figure 3E) and control or between AMI and CAD (Figure 4D). To further analyze the core mRNA of light-yellow modules, the top 50 exosomal mRNAs of the topological overlap matrix in this module were used to construct a network diagram (Figure 5E). In this network, the exosomal mRNA (with eigengene connectivity $>0.8$ ) in the lightyellow module was considered as core mRNA and was labored with yellow (Figure 5E). Thus, six mRNAs including ALPL, CXCR2, ELL2, EMC9, FAM129A, and DBF4B were identified as core mRNA.

\section{Potential clinical value of exosomal ALPL and CXCR2}

To estimate the potential clinical value of the six core exosomal mRNAs, ROC curve analysis was applied (Figure 6A). The AUC indicated that there were two mRNAs with great predictive accuracy (ALPL (AUC: 0.99); CXCR2 (AUC: 0.98)). The correlation analysis between these two exosomal mRNAs and clinical baseline characteristics (Figure 6B) indicated that ALPL and CXCR2 were associated with the neutrophil count ( $R=0.52, R=0.51$, respectively). Statistical analysis of expression suggested that exosomal mRNA ALPL and CXCR2 from the AMI plasma were significantly higher than the other groups (Figure 6C, 6D). Besides, exosomal ALPL in the CAD group was also significantly higher than the control group. It might suggest that circulating exosomal ALPL would extremely increase with the progression of coronary plaque to acute myocardial infarction.

Since most results of the analysis of exosomal mRNAs in AMI patients mainly pointed to acute inflammation respond mediated by neutrophils, we tried to apply the PCA with neutrophil count and neutrophil ratio to identify the three groups. The PCA of neutrophil count and neutrophil ratio showed that although the AMI group could separate from the other groups, there was much overlap between CAD and control (Figure 6E). However, when we applied PCA with the expression value of ALPL and CXCR2, the three groups could separate from each other (Figure 6F). To sum up, besides the potential of great predictive accuracy, these two exosomal ALPL and CXCR2 might also be good for predictive identification.

\section{Discussion}


Recent years have witnessed the great advances in exosomal microRNAs in cardiovascular disease study but very few studies revealed the features and potential clinical value of exosomal mRNAs. In this study, we found that the plasma exoLRs largely consisted of mRNAs. The alteration of these mRNAs in AMI patients could indicate the neutrophilic inflammation of the circulatory system. Among these mRNAs, we found ALPL and CXCR2 with good predictive accuracy might be potential biomarkers for AMI diagnosis.

Our exoLRs sequencing data from this study suggested that the plasma exoLRs were mainly mRNAs. It is not surprising to find the exosomal circRNAs and IncRNAs were expressed in low abundance since they had specific spatiotemporal expression patterns [22-24]. A relatively high number of long RNAs were identified in AMI samples. On the one hand, the AMI samples in this study were from relatively aged patients. Previous studies demonstrated that aging was a profound factor in changes of circulating extracellular vesicles in concentration, size, and cargo $[25,26]$. On the other hand, stress including hypoxia, inflammation or injury could induce cardiomyocytes or other cells to secret exosomes[27].

To provide a better indication of the change exosomal mRNAs in the AMI patients, we applied bioinformatics approaches to analyze different exosomal mRNAs by comparing to CAD patients and healthy individuals. Besides, we also used co-expression network analysis to investigate the exosomal mRNAs in AMI. The results of our analyses all indicated that the acute inflammatory response mediated by neutrophils might be the core biological processes in the AMI patients. These findings were consistent with the current AMI studies. Since the cardiomyocyte is extremely sensitive to ischemic injury, the reduced blood supply to the myocardium would initially cause the injury and further lead to an intense inflammatory response once the AMI occurred $[28,29]$. Furthermore, Neutrophil extracellular traps were identified as the crucial triggers and structural factors of various forms of thrombosis[30], besides, neutrophilic inflammation was found to influence the infarct size, healing, and cardiac function after myocardial infarction[31]. Although the exact physiological role of exosome in AMI is still poorly understood, inflammation-related alterations in exosomal RNAs were associated with the biological processes of AMI.

Since miscellaneous immune cells would take parts in cardiac repair during different phases of cardiovascular disease[32], neutrophils were traditionally viewed as biomarkers [33]. Similarly, we found two exosomal mRNA ALPL and CXCR2 might be served as potential diagnostic biomarkers for AMI as they were specifically enriched in circulating neutrophils which could be confirmed by the Human Protein Atlas (http://www.proteinatlas.org) [34].

The previous study indicated that ALPL could regulate the cardiac fibrosis during myocardial infarction through TGF- $31 /$ Smads and P53 signaling pathways [35]. Similarly, the inhibitor of ALPL was found to be a potential target to treat cardiovascular disease by attenuating the arterial calcification in a non-chronic kidney disease context [36]. Consistent with our study, we found the expression level of exosomal mRNA ALPL in the AMI samples was the highest, CAD the next and healthy individuals' samples the lowest. Whether exosomal mRNA ALPL would increase during the progression of coronary plaque to acute myocardial infarction still need to be further studied. 
CXCR2, an intriguing biomolecule had dual effects on the myocardial ischemia-reperfusion injury for myocardial damage and cardioprotective effect $[37,38]$. Nonetheless, increasing evidence showed that CXCR2 might play a crucial cardioprotective role in myocardial infarction through enhancing myeloid progenitor production and upregulating cardiac adhesion molecules [31, 39]. Our data showed that the circulating exosomal mRNA CXCR2 was a potential biomarker for AMI with high diagnostic efficiency but the mechanism of secreting the exosomal mRNA CXCR2 need to be further studied.

Until recently, circulating plasma exosomes were shown to interact with a variety of cell types and tissue. However, the exact physiological role and function of plasma exosomal mRNAs are poorly understood. Whether the plasma exosomal mRNAs would be functional mainly depended on they were intact or fragments. In fact, some exosomal mRNAs were found to be full length and functional[40]. Nevertheless, further investigations of these exosomal mRNAs were still needed. Although few studies focused on the exosomal mRNA of AMI, it should be noted that these findings still needed to be validated in prospective clinical trials as the sample size was not big.

\section{Conclusions}

In summary, our study explored the alteration of exoLRs in the AMI patients which might associate with the acute inflammatory response mediated by neutrophils. Moreover, we found that exosomal mRNAs ALPL and CXCR2 might be potentially useful for AMI diagnosis.

\section{Abbreviations}

exoLRs: exosomal long RNAs; AMI: acute myocardial infarction; CAD: coronary artery disease; mRNAs: messenger RNAs; ROC: receiver operating characteristic curve; circRNA: circular RNA; IncRNA: Iong noncoding RNA; ESC: european society of cardiology; EDTA: ethylene diamine tetraacetic acid; TEM: transmission electron microscopy; GO: gene ontology; BP: biological processes; CC: cellular component; MF: molecular function; TOM: topological overlap matrix; PCA: principal component analysis; SD: standard deviation; FPKM: fragments per kilobase per million; AUC: under the curve; WGCNA: weighted gene co-expression network analysis; MONO: absolute monocyte count; MONO\%: the percentage of monocyte; NEUT: absolute neutrophil count; NEUT\%: the percentage of neutrophil; CK-MB : creatine kinase, MB Form; HBDH : hydroxybutyrate-dehydrogenase.

\section{Declarations}

\section{Ethics approval and consent to participate}

The authors declare no potential conflict of interest. The ethical approval of human sample collection was achieved from the Ethics Committee of Guangdong Provincial People's Hospital (No.2018160A), and all patients provided informed consent. All procedures were conformed with the Helsinki Declaration of 
1975 (as revised in 2008) concerning Human Rights, and followed out policy concerning Informed Consent as shown on Springer.com.

\section{Consent for publication}

Not applicable.

\section{Availability of data and materials}

The RNA-Seq data used to support the findings of this study are available from the corresponding author on reasonable request.

\section{Competing interests}

The authors declare that they have no competing interests.

\section{Funding}

This work was supported by the Natural Science Foundation of Guangdong Province (No. 2020A1515010738), Science and Technology Plan Program of Guangdong Province (No. 2017B030314041), Science and Technology Plan Program of Guangzhou (No.201604020143, No.201604020018, No.201604020186, No.201510010254, and No.201803040012), and the National Key Research and Development Program of China (No.2017YFC1307603, No.2016YFC1301305), the Key Area R\&D Program of Guangdong Province ( No.2019B020227005).

\section{Authors' contributions}

GDH and YQW coordinated the testing of samples and performed the bioinformatics analyses. LL, JYH and KL performed the statistical analyses. YLY and CLC collected the samples. BZ and YQF designed the original study and drafted the manuscript. All authors critically reviewed and approved the fnal manuscript.

\section{Acknowledgments}


We appreciate Guangzhou Epibiotek Co., Ltd for excellent technical assistance.

\section{References}

1. vN G. G DA, G R: Shedding light on the cell biology of extracellular vesicles. Nature reviews Molecular cell biology. 2018;19:213-28.

2. OPB W, MÁ B, XO JL. B, S EA: Advances in therapeutic applications of extracellular vesicles. Science translational medicine 2019, 11.

3. M VCDS, ML MTS, lacobellis ZGW. G \%J Nutrition m, NMCD cd: Novel atherogenic pathways from the differential transcriptome analysis of diabetic epicardial adipose tissue. 2017, 27:739-750.

4. R S, T P, JE F: Circulating Extracellular Vesicles in Human Disease. The New England journal of medicine 2018, 379:958-966.

5. D T. H H, X F, W H: Mesenchymal stem cells-derived exosomal microRNAs contribute to wound inflammation. Science China Life sciences. 2016;59:1305-12.

6. CM B, PE XL. R, N A: Extracellular vesicles in coronary artery disease. Nature reviews Cardiology. 2017;14:259-72.

7. C JZSLLLML, J G. Y, S M: Exosome and exosomal microRNA: trafficking, sorting, and function. Genom Proteom Bioinform. 2015;13:17-24.

8. KM K, K A MM. D K, M G: RNA in extracellular vesicles. Wiley interdisciplinary reviews RNA 2017, 8.

9. H RG F, one EEJP: Network analysis of inflammatory responses to sepsis by neutrophils and peripheral blood mononuclear cells. 2018, 13:e0201674.

10. LL L, WJ YFYW, HF W, ZL N, LT L, JD ZBW, SD Z. C, BC L: Exosomal CCL2 from Tubular Epithelial Cells Is Critical for Albumin-Induced Tubulointerstitial Inflammation. Journal of the American Society of Nephrology: JASN. 2018;29:919-35.

11. MD LDWLPQ, WW XXWSNDH W, C T, W S, et al: Circulating Long RNAs in Serum Extracellular Vesicles: Their Characterization and Potential Application as Biomarkers for Diagnosis of Colorectal Cancer. Cancer epidemiology, biomarkers \& prevention: a publication of the American Association for Cancer Research, cosponsored by the American Society of Preventive Oncology 2016, 25:11581166.

12. JL A. DA M: Acute Myocardial Infarction. N Engl J Med. 2017;376:2053-64.

13. B I SJ, C B-D SAMJA, ALP HB, JA CFC. G, S H, et al: 2017 ESC Guidelines for the management of acute myocardial infarction in patients presenting with ST-segment elevation: The Task Force for the management of acute myocardial infarction in patients presenting with ST-segment elevation of the European Society of Cardiology (ESC). European heart journal. 2018;39:119-77.

14. G M, U S, C SAFA, C AABRBFCTC. DM, et al: 2013 ESC guidelines on the management of stable coronary artery disease: the Task Force on the management of stable coronary artery disease of the European Society of Cardiology. European heart journal. 2013;34:2949-3003. 
15. D K, B L, SL S: HISAT: a fast spliced aligner with low memory requirements. Nature methods 2015, 12:357-360.

16. X Y. TO C: Acfs: accurate circRNA identification and quantification from RNA-Seq data. Scientific reports. 2016;6:38820.

17. B Z YZ, AH LPMC, O K, C T. B, communications CSJN: Metascape provides a biologist-oriented resource for the analysis of systems-level datasets. 2019, 10:1523.

18. G Y, LG W. Y H, QY H: clusterProfiler: an R package for comparing biological themes among gene clusters. Omics: a journal of integrative biology. 2012;16:284-7.

19. P L, S H: WGCNA: an R package for weighted correlation network analysis. BMC bioinformatics 2008 , 9:559.

20. D O, JH M, AR JB. P, B D: Cytoscape Automation: empowering workflow-based network analysis. Genome biology. 2019;20:185.

21. NN P, TG VS, M B, MJ IGR, C G, LT HVL. U, J H, et al: Genome-wide changes in IncRNA, splicing, and regional gene expression patterns in autism. Nature. 2016;540:423-7.

22. F M-H SB. Emerging role of non-coding RNA in oral cancer. Cellular signalling. 2018;42:134-43.

23. X LY, X Z PYL, Q YMCW. F, S W, H L, J L: Long non-coding RNA HOTAIR promotes exosome secretion by regulating RAB35 and SNAP23 in hepatocellular carcinoma. Mol Cancer. 2019;18:78.

24. P PG. P, N R: circBase: a database for circular RNAs. RNA. 2014;20:1666-70.

25. FJ A, A FL, PV D, T B-M Y. A B, L W, D G, S M, SH L, et al: Cellular senescence contributes to agedependent changes in circulating extracellular vesicle cargo and function. Aging cell. 2020;19:e13103.

26. C M-B CB, J S-R, A R-D, L G-M MI. J G, J V: Extracellular vesicles and redox modulation in aging. Free Radic Biol Med. 2020;149:44-50.

27. DA C, AN O. YV B: Cardiac Extracellular Vesicles in Normal and Infarcted Heart. International journal of molecular sciences 2016, 17.

28. JM V, C SN, TJ VDC, M G-R, LA CDNB, DR J, CA G, DG C, PR J. R: The cardiac lymphatic system stimulates resolution of inflammation following myocardial infarction. J Clin Investig. 2018;128:3402-12.

29. S T, A A: The NLRP3 inflammasome in acute myocardial infarction. Nature reviews Cardiology 2018, 15:203-214.

30. JB M, B H-T-N. Thrombi and neutrophils. Circulation research. 2015;116:1107-8.

31. MJ S, C MHKNJDMDKBCS, O W. S, S S: The time-of-day of myocardial infarction onset affects healing through oscillations in cardiac neutrophil recruitment. EMBO molecular medicine. 2016;8:937-48.

32. A K, A S, K S, Y K, molecular NHJJo, cardiology c: Matrix metalloproteinase-12 produced by Ly6C macrophages prolongs the survival after myocardial infarction by preventing neutrophil influx. 2019, $131: 41-52$. 
33. C S-R QB. A O-G, Cardiology SOJNr: Neutrophils as regulators of cardiovascular inflammation. 2020.

34. K FP. J, M U: The Human Protein Atlas-a tool for pathology. J Pathol. 2008;216:387-93.

35. LY LG, SY WZQLDJ, YQ W, HM G, LN TMS. Y, S Q, et al: TNAP inhibition attenuates cardiac fibrosis induced by myocardial infarction through deactivating TGF- $\beta 1 /$ Smads and activating P 53 signaling pathways. Cell death disease. 2020;11:44.

36. B O, PC DH. A V: Inhibition of tissue non-specific alkaline phosphatase; a novel therapy against arterial media calcification? J Pathol. 2020;250:248-50.

37. ST T, W M, SM KMLL, Circulation FJWB. KRJ: Opposing effects mediated by the chemokine receptor CXCR2 on myocardial ischemia-reperfusion injury: recruitment of potentially damaging neutrophils and direct myocardial protection. 2003, 108:2387-2392.

38. EA L, I K, TT SKAKSS, Weber SRBJB. C \%J Arteriosclerosis t, biology v: Compartmentalized protective and detrimental effects of endogenous macrophage migration-inhibitory factor mediated by CXCR2 in a mouse model of myocardial ischemia/reperfusion. 2013, 33:2180-2186.

39. JM A, C DF, MI GC, M C-A, LA C, H H-E W, C S-R JR. I C, et al: A Neutrophil Timer Coordinates Immune Defense and Vascular Protection. 2019, 50:390-402.e310.

40. $H V, K E, A B, M S, J J ~ L$, biology LJJNc: Exosome-mediated transfer of mRNAs and microRNAs is a novel mechanism of genetic exchange between cells. 2007, 9:654-659.

\section{Table 1}

Table.1 Clinical baseline characteristics of patients 


\begin{tabular}{|c|c|c|c|}
\hline & AMI & $\mathrm{CAD}$ & CONTROL \\
\hline & $\mathrm{n}=10$ & $\mathrm{n}=8$ & $\mathrm{n}=10$ \\
\hline \multicolumn{4}{|l|}{ Gender } \\
\hline Male & 8 & 7 & 2 \\
\hline Female & 2 & 1 & 8 \\
\hline Age(years) & $62.80 \pm 10.04^{*}$ & $53.88 \pm 9.52$ & $47.00 \pm 14.02$ \\
\hline Diabetes & 4 & 3 & 0 \\
\hline \multicolumn{4}{|l|}{ Hypertension } \\
\hline Grade 1 & 1 & 0 & 0 \\
\hline Grade 2 & 1 & 2 & 0 \\
\hline Grade 3 & 2 & 4 & 0 \\
\hline Current smoking & 3 & 0 & 0 \\
\hline Current drinking & 2 & 0 & 0 \\
\hline MONO & $1.01 \pm 0.36^{*}$ & $0.55 \pm 0.26$ & $0.44 \pm 0.09$ \\
\hline MONO\% & $8.46 \pm 3.70$ & $8.29 \pm 2.42$ & $7.24 \pm 0.78$ \\
\hline NEUT & $10.27 \pm 3.62^{* * \# \#}$ & $4.03 \pm 1.80$ & $3.96 \pm 1.90$ \\
\hline NEUT\% & $78.00 \pm 12.72^{* * \# \#}$ & $59.88 \pm 8.01$ & $59.58 \pm 11.48$ \\
\hline CK-MB(U/L) & $235.47 \pm 302.70^{* \#}$ & $11.80 \pm 2.21$ & $10.4 \pm 0.64$ \\
\hline $\mathrm{HBDH}(\mathrm{U} / \mathrm{L})$ & $699.00 \pm 670.98^{* \#}$ & $89.00 \pm 12.29$ & $97.30 \pm 13.21$ \\
\hline
\end{tabular}

Results are presented as the mean $\pm \mathrm{SD}$. (*: the AMI group compared to control group $* \mathrm{P}<0.05, * * \mathrm{P}<0.01$; \#: the AMI group compared to CAD group, ${ }^{\#} \mathrm{P}<0.05,{ }^{\# \#} \mathrm{P}<0.01 ;$ MONO: absolute monocyte count; MONO\%: the percentage of monocyte; NEUT: absolute neutrophil count; NEUT\%: the percentage of neutrophil; CK-MB : creatine kinase, MB Form; HBDH : hydroxybutyrate-dehydrogenase) 


\section{Figures}
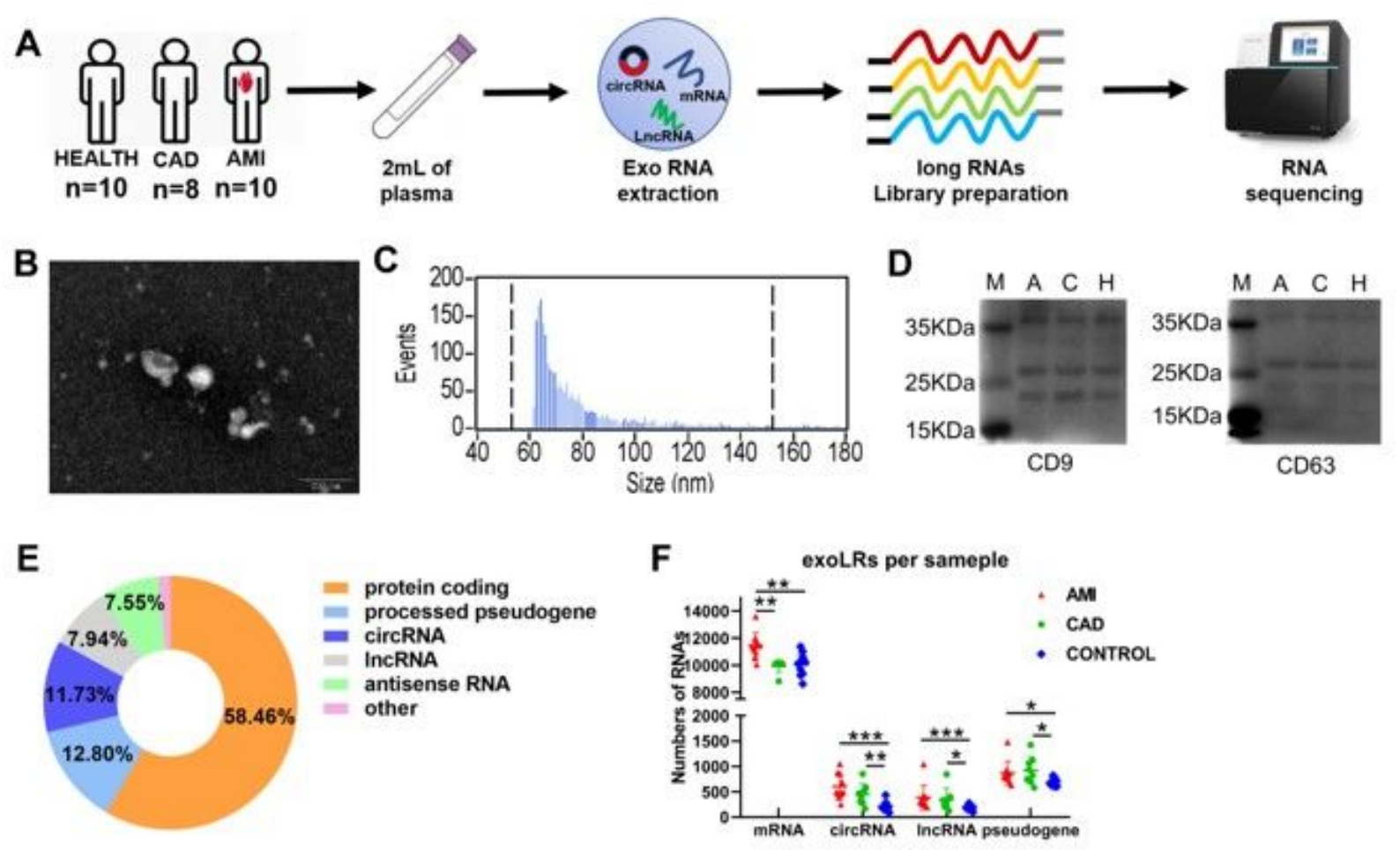

\section{Figure 1}

A brief view of the workflow of human plasma exosomal long RNA-seq and its characteristics in each group. A: Work flow of exosomal long RNA-seq of human plasma. B囚Electron microscopy image of isolated exosomes. C: Size distribution measurements of isolated exosomes. D: Western blot analysis of exosomal markers. (M: marker; A: acute myocardial infarction; C: coronary artery disease; $\mathrm{H}$ : healthy individuals) E: Distribution of mapped reads to the genes with annotation and identified circRNA. F: The number of exoLRs for each group displayed by scatterplot. Results are described as the mean $\pm \mathrm{SD}$ ( ${ }^{\star} \mathrm{P}<$ $0.05,{ }^{*} \mathrm{P}<0.01,{ }^{* \star *} \mathrm{P}<0.001$; Exo RNA: exosomal RNA; exoLRs: exosomal long RNAs). 
A

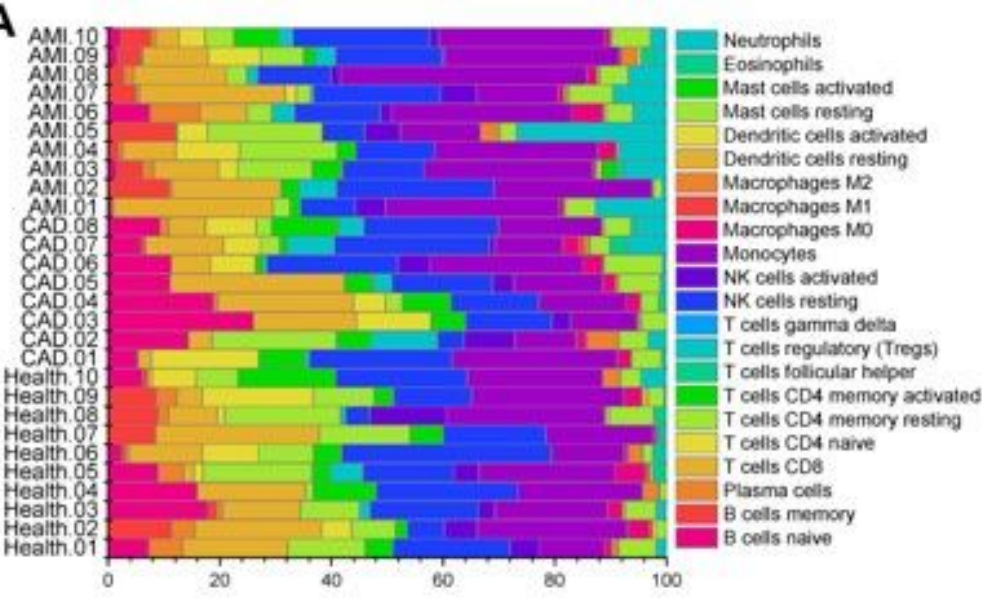

C

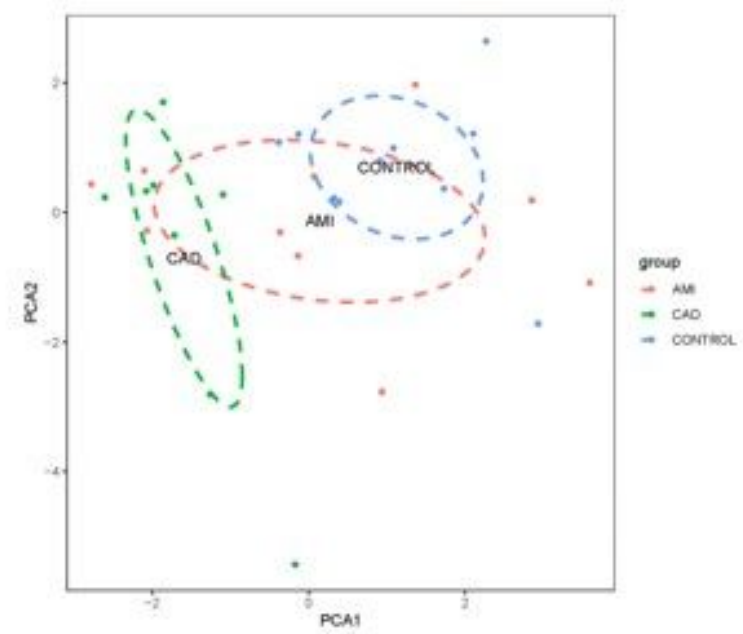

B

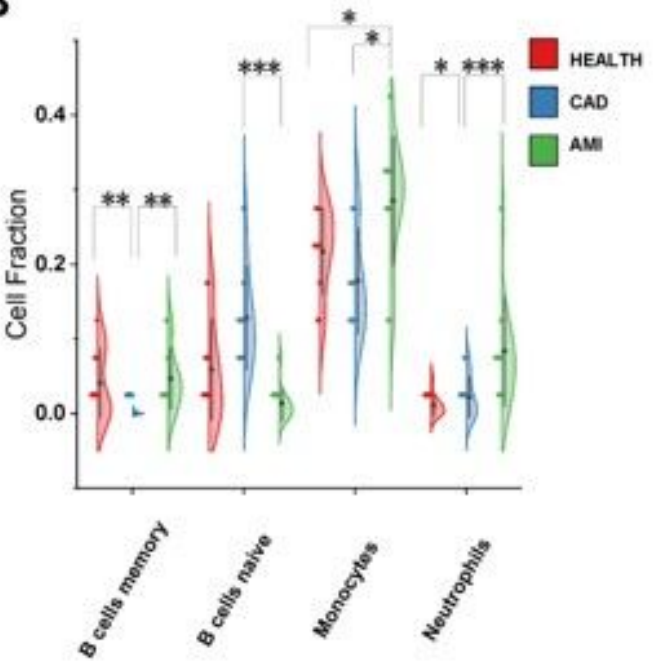

Figure 2

The Exosomal long RNA reflected relative fractions of different immune cell types. A: Relative fraction of twenty-two types of immunocyte assessed from exosomal long RNA-seq data by CIBERSORT. B: Violin plot of the fraction of four types of immune cells. Results are presented as the mean $\pm S D\left({ }^{*} P<0.05\right.$, $* * P$ $<0.01, * \star \star P<0.001)$ C: The PCA of all types of immune cells. 
A

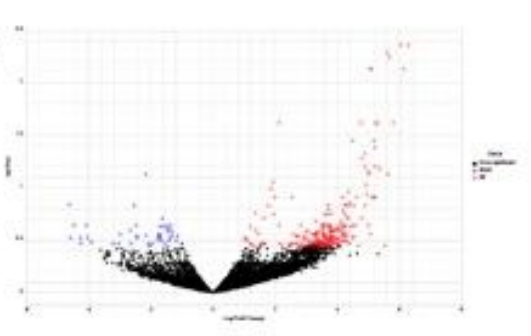

C

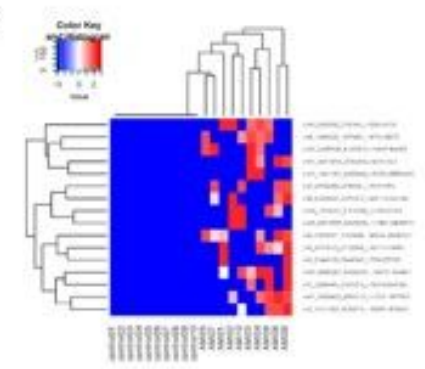

D

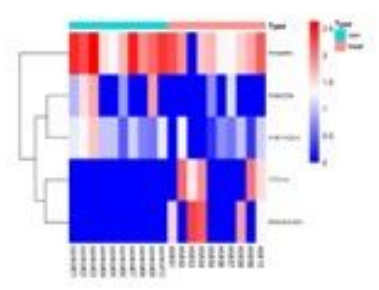

B

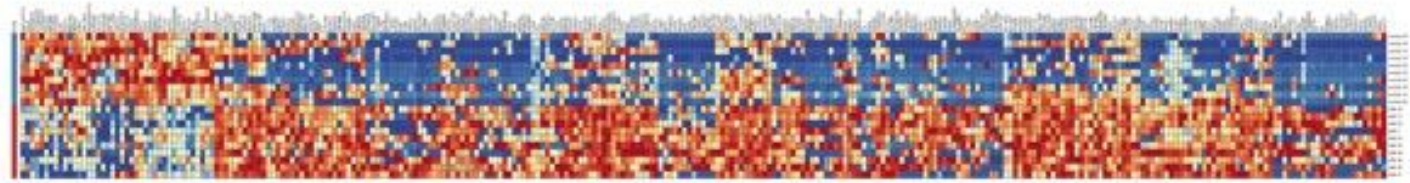

E

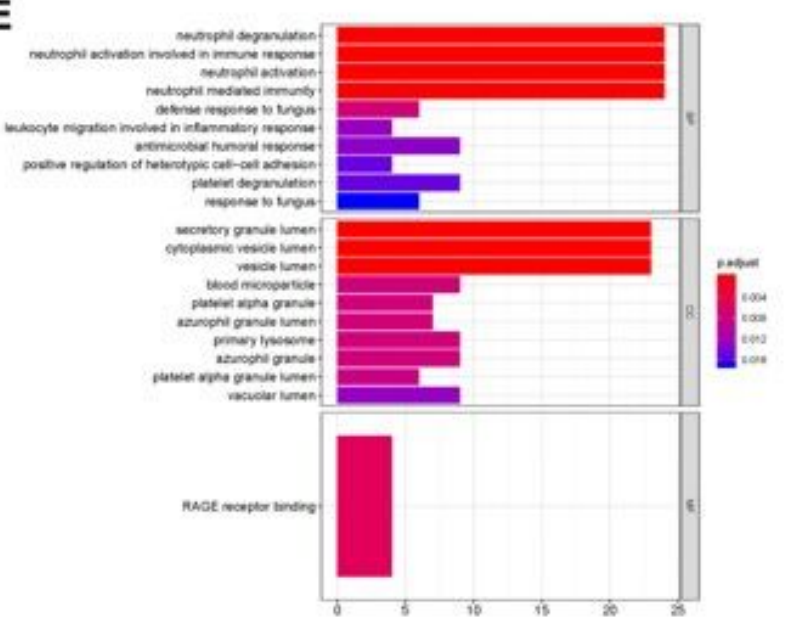

$\mathbf{F}$
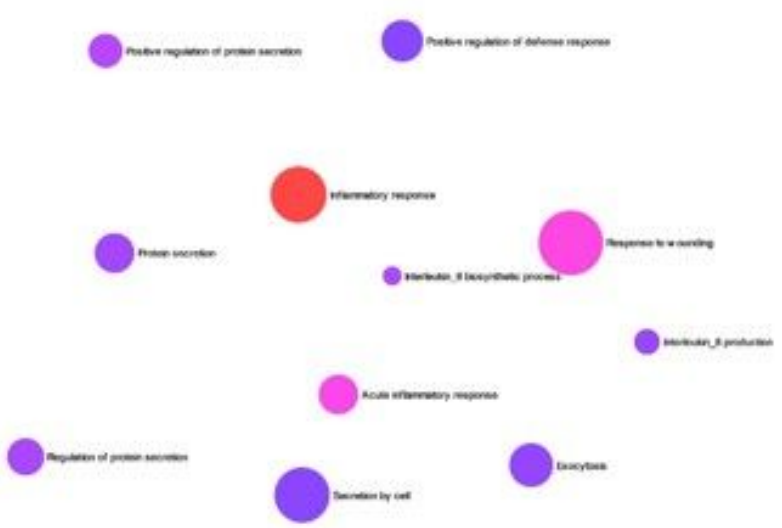

\section{Figure 3}

Different exosomal long RNA analysis between the AMI and the control. A: Volcano plots of different exosomal mRNAs analysis between AMI and control. B: Heatmap of significant mRNA between AMI and control. C: Heatmap of significantly IncRNA between AMI and control. D: Heatmap of significantly circRNA between AMI and control. E and F: Functional enrichment analysis of significantly different exosomal mRNA. 
A

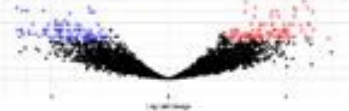

B

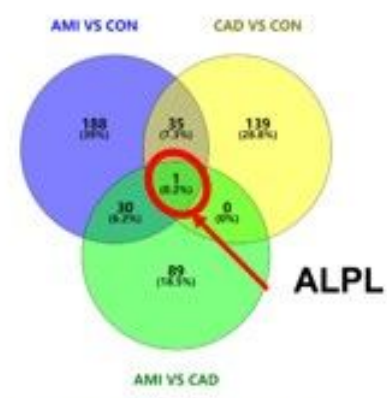

C

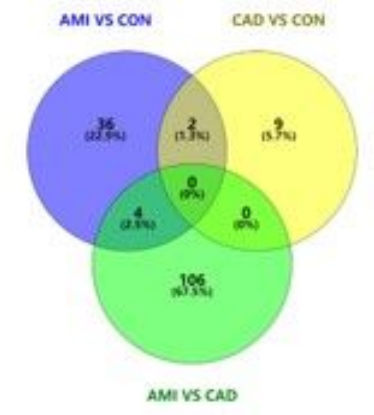

E

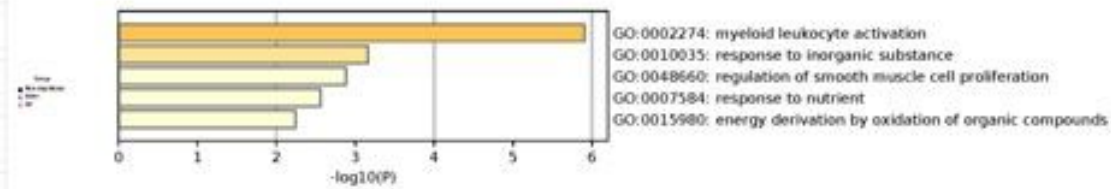

D

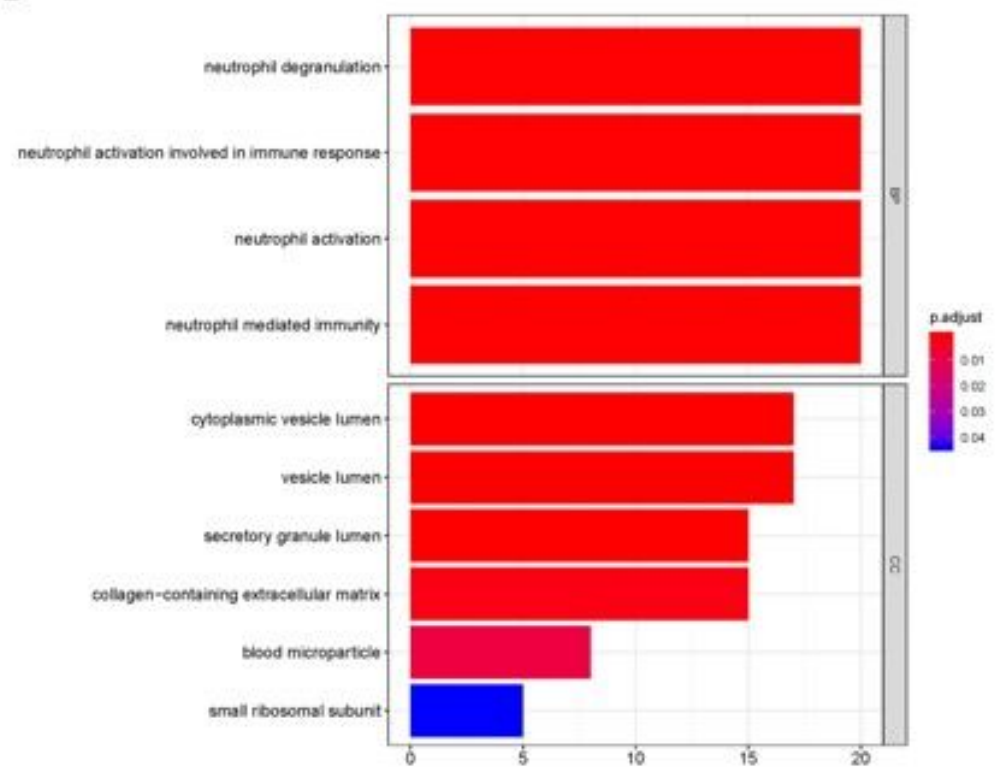

\section{Figure 4}

Different exosomal long RNA analysis between AMI and CAD. A: Volcano plots of different exosomal mRNAs analysis between AMI and CAD group. B-C: Comparation of differently up- or down-regulated exosomal mRNAs between AMI and control, AMI and CAD, and CAD and control. These three comparison sets were intersected with each other. D囚Functional enrichment analysis of significantly different exosomal mRNA between AMI and CAD. E: Gene ontology analysis result of 35 different exosomal mRNAs between the comparison of AMI and control and comparison of AMI and CAD. 


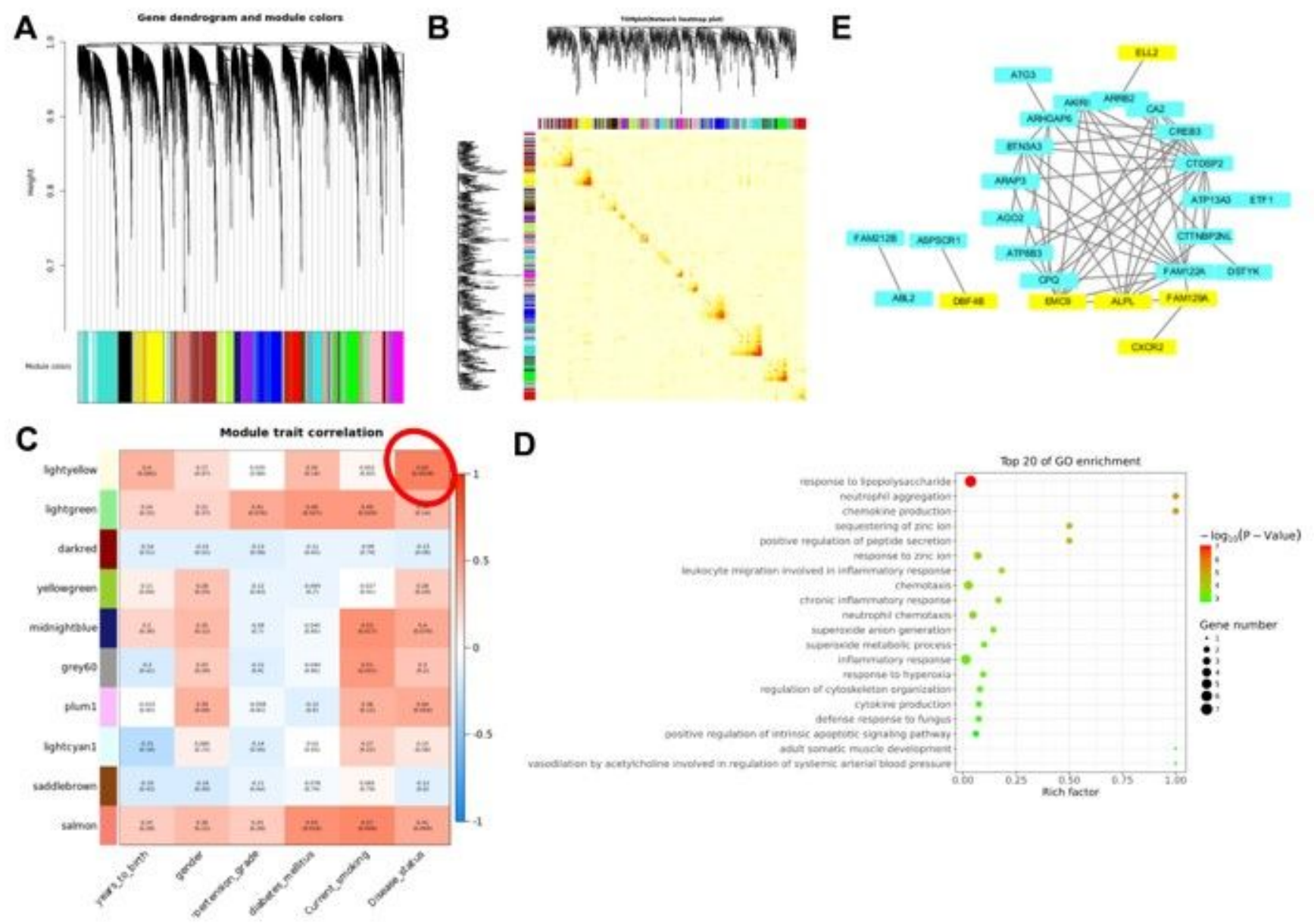

Figure 5

Co-expression network analysis of exosomal mRNAs in AMI. A: Cluster diagram showing co-expression modules identified by WGCNA. B: The heat map plot showed the topological overlap matrix (TOM) among all mRNAs. Light color shows low overlap, and red color indicates a higher overlap. The left side and the top side show the gene dendrogram and module assignment. C: Heatmap of modules-trait relationship. D: GO enrichment analysis of light-yellow modules. E: Network visualization of the top 50 exosomal mRNAs of the topological overlap matrix in light-yellow modules. The exosomal mRNA with eigengene connectivity $>0.8$ were highlighted in yellow color. 

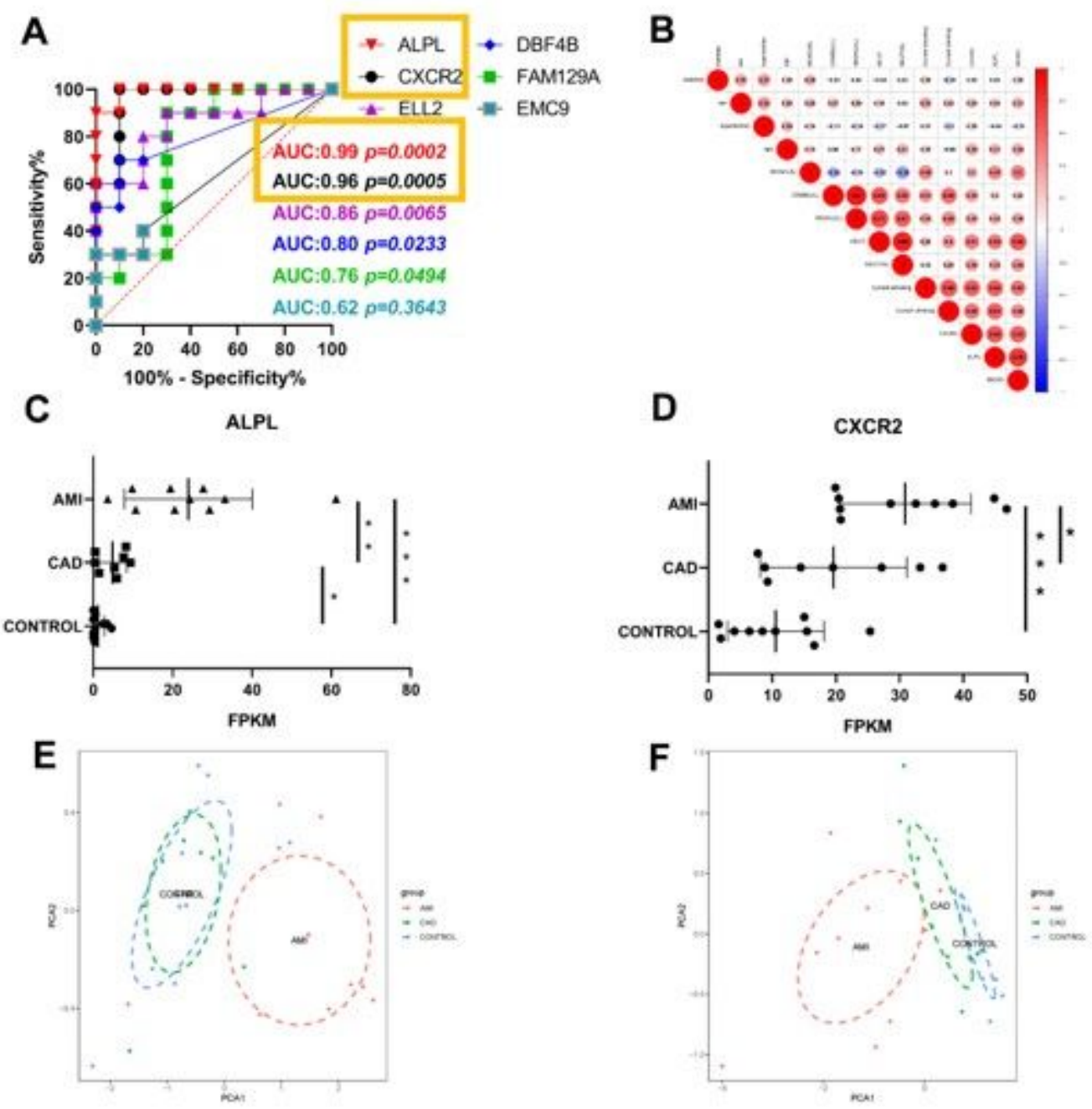

\section{Figure 6}

Potential clinical value of ALPL and CXCR2. A: The ROC curve analysis of six core exosomal mRNAs. B: The correlation analysis between these two exosomal mRNAs and clinical baseline characteristics. C-D: The statistical analysis of expression of ALPL and CXCR2 among AMI, CAD, and control group. Results are presented as the mean $\pm S D\left(* P<0.05,{ }^{*} P<0.01\right.$, $\left.{ }^{\star} * \star P<0.001\right) E$ : PCA of neutrophil count and neutrophil ratio. F: PCA of the expression of ALPL and CXCR2. 